\title{
A SHARP BERNSTEIN-TYPE THEOREM FOR ENTIRE MINIMAL GRAPHS
}

\author{
Alberto Farina \\ LAMFA, CNRS UMR 7352 \\ Université de Picardie Jules Verne \\ 33 rue Saint-Leu, 80039 Amiens, France
}

\begin{abstract}
We consider entire solutions $u$ to the minimal surface equation in $\mathbb{R}^{N}$, with $N \geq 8$, and we prove the following sharp result : if $N-7$ partial derivatives $\frac{\partial u}{\partial x_{j}}$ are bounded on one side (not necessarily the same), then $u$ is necessarily an affine function.
\end{abstract}

MSC: 53A10, 58JO5, 35J15

\section{Introduction and main results}

The graph of a smooth function $u: \mathbb{R}^{N} \rightarrow \mathbb{R}$ is a minimal surface in $\mathbb{R}^{N+1}$ if and only if $u$ is a solution to the minimal surface equation

$$
-\operatorname{div}\left(\frac{\nabla u}{\sqrt{1+|\nabla u|^{2}}}\right)=0 \quad \text { in } \quad \mathbb{R}^{N}, \quad N \geq 2 .
$$

In his work [2] (see also [10]) S.N. Bernstein proved that any smooth solution $u$ to the minimal surface equation in $\mathbb{R}^{2}$ must be an affine function. This result has been extended to $\mathbb{R}^{3}$ by E. De Giorgi [6], to $\mathbb{R}^{4}$ by J.F. Almgren [1] and, up to dimension $N=7$, by J. Simons [16]. On the other hand, in the celebrated paper [3], E. Bombieri, E. De Giorgi and E. Giusti proved the existence of a non-affine entire solution of the minimal surface equation (1.1) for any $N \geq 8$. Nevertheless, J. Moser [15] was able to prove that, if $\nabla u$ is bounded on $\mathbb{R}^{N}$, then $u$ must be again an affine function, and this result holds true for every dimension $N \geq 2$. Later, E. Bombieri and E. Giusti [5] generalized Moser's result by assuming that only $N-1$ partial derivatives of $u$ are bounded on $\mathbb{R}^{N}$, $N \geq 2$. In the recent work [7] the author has further improved this result. More precisely, in [7] it is demonstrated that any smooth solution $u$ to the minimal surface equation (1.1) with $N-1$ partial derivatives bounded on one side (not necessarily the same) is necessarily an affine function.

The main result of the present work is the following 
Theorem 1.1. Assume $N \geq 8$ and let $u$ be a solution of the minimal surface equation

$$
-\operatorname{div}\left(\frac{\nabla u}{\sqrt{1+|\nabla u|^{2}}}\right)=0 \quad \text { in } \quad \mathbb{R}^{N} .
$$

If $N-7$ partial derivatives of $u$ are bounded on one side (not necessarily the same) then $u$ is an affine function.

Theorem 1.1 is sharp. Indeed, it cannot hold true if one assumes that only $N-8$ partial derivatives are bounded on one side. To see this, let us denote by $U=U\left(x_{1}, \ldots, x_{8}\right)$ the non-affine solution of (1.2) in $\mathbb{R}^{8}$ constructed in [3] and, for every $N \geq 8$, set $u_{N}\left(x_{1}, \ldots, x_{N}\right):=U\left(x_{1}, \ldots, x_{8}\right)$. Clearly, $u_{N}$ solves (1.2), it has $N-8$ partial derivatives that are identically zero, but $u_{N}$ is not an affine function.

The proof of the previous theorem is based on the following result, which is interesting in its own.

Theorem 1.2. Let $u$ be a non-affine solution of the minimal surface equation

$$
-\operatorname{div}\left(\frac{\nabla u}{\sqrt{1+|\nabla u|^{2}}}\right)=0 \quad \text { in } \quad \mathbb{R}^{N}, \quad N \geq 2
$$

such that

$$
u(0)=0
$$

Suppose also that there exists an integer $k \in\{1, \ldots, N\}$ such that, for every $\alpha=1, \ldots, k$, the partial derivative $\frac{\partial u}{\partial x_{\alpha}}$ is bounded from below on $\mathbb{R}^{N}$.

Then, any blow-down $C \subset \mathbb{R}^{N+1}$ associated to the subgraph of $u$ is a minimal cylinder of the form

$$
C=P \times \mathbb{R}=\left(\mathbb{R}^{k} \times P^{\prime}\right) \times \mathbb{R},
$$

where $P^{\prime} \subset \mathbb{R}^{N-k}$ is a minimal cone singular at the origin.

\section{Proofs}

Let us first consider Theorem 1.2.

Proof of Theorem 1.2. Let $U$ be the subgraph of $u$ and $U_{j}$ the one of the functions $u_{j}(x)=\frac{u(j x)}{j}$, where $x \in \mathbb{R}^{N}$ and $j \geq 1$.

By assumption, $U$ is a non-trivial set of least perimeter in $\mathbb{R}^{N+1}$ with $0 \in \partial U$. Therefore, a classical procedure (see e.g. Theorem 17.3 and Theorem 9.3 of [8]) provides a minimal cone $C \subset \mathbb{R}^{N+1}$ (with vertex at the origin), as the limit of a subsequence of $U_{j}$ (still denoted by $U_{j}$ ) with respect to the $L_{l o c}^{1}$ convergence. The minimal cone $C$ is usually called a blow-down of $U$ and we have that, for almost every $R>0$, 


$$
\omega_{N} \leq R^{1-(N+1)} \int_{B(0, R)}\left|D \mathbf{1}_{C}\right| .
$$

where $\omega_{N}$ denotes de volume of the unit ball of $\mathbb{R}^{N}$. Indeed, by the minimality of the sets $U_{j}$, the monotonicity of the functions $R \rightarrow R^{1-(N+1)} \int_{B(0, R)}\left|D \mathbf{1}_{U_{j}}\right|$ and the fact that $0 \in \partial U_{j}$, the following well-known density estimates hold (see for instance formula (5.16) on p. 72 of [8] or formula (1.13) on p.2 of [9])

$$
\omega_{N} \leq(R j)^{1-(N+1)} \int_{B(0, R j)}\left|D \mathbf{1}_{U}\right|=R^{1-(N+1)} \int_{B(0, R)}\left|D \mathbf{1}_{U_{j}}\right|
$$

Therefore

$$
\omega_{N} \leq R^{1-(N+1)} \int_{B(0, R)}\left|D \mathbf{1}_{U_{j}}\right| \rightarrow R^{1-(N+1)} \int_{B(0, R)}\left|D \mathbf{1}_{C}\right|,
$$

proving (2.1). From (2.1) we get that $0 \in \partial C$ and, by Lemma 16.3 and Proposition 16.5 of [8], we also know that $C$ is itself a subgraph of a generalized solution to the minimal surface equation $v: \mathbb{R}^{N} \rightarrow[-\infty,+\infty]$ (also called quasi-solution to the minimal surface equation) (see [13], [11], [8]).

Also the set

$$
P=\left\{x \in \mathbb{R}^{N}: v(x)=+\infty\right\}
$$

must be non-empty since $u$ is non-affine. To see this we follow [8]. If $P=\emptyset$ then, by Lemma 17.7 of [8], the family of functions $u_{j}$ is equibounded from above on compact sets of $\mathbb{R}^{N}$. The latter and the definition of $u_{j}$ immediately provides that

$$
\sup _{B(0, j)} u \leq \mathcal{K} j
$$

for some constant $\mathcal{K}>0$. On the other hand, the celebrated gradient estimate of [4] tells us that

$$
\forall x \in \mathbb{R}^{N}, \forall R>0, \quad|\nabla u(x)| \leq C_{1} \exp \left[C_{2}\left(\frac{\sup _{B(x, R)} u-u(x)}{R}\right)\right]
$$

where $C_{1}$ and $C_{2}$ are positive constant depending only on the dimension $N$. Now, combining (2.5),(2.6) and letting $j \rightarrow+\infty$ we obtain that $|\nabla u| \in L^{\infty}\left(\mathbb{R}^{N}\right)$. Thus, we can apply the result of Moser [15], already recalled in the introduction, to get that $u$ is an affine function. The latter is impossible since we are supposing that $u$ is not affine.

We also remark that

$$
P \times \mathbb{R} \subset C
$$

by construction, and so $P \not \equiv \mathbb{R}^{N}$, since $C \not \equiv \mathbb{R}^{N+1}$.

Also, $P$ is a minimal cone in $\mathbb{R}^{N}$, with vertex at the origin (since $C$ is a minimal cone with vertex at the origin). Combining the two latter pieces of information we also get that the origin of $\mathbb{R}^{N}$ belongs to $\partial P$ and so $0 \in \partial(P \times \mathbb{R})$. 
Next we observe that $P$ is singular at the origin of $\mathbb{R}^{N}$. Suppose not, then $P$ would be a half-space of $\mathbb{R}^{N}$ and thus the minimal cone $C$ would contain the half-space $P \times \mathbb{R}$ which, in turn, would give $C \equiv P \times \mathbb{R}$ (see for instance Theorem 15.5 of [8] or [12]) and so

$$
\forall R>0 \quad \omega_{N}=R^{1-(N+1)} \int_{B(0, R)}\left|D \mathbf{1}_{C}\right|
$$

Therefore, by (2.8), the monotonicity of $R \rightarrow R^{1-(N+1)} \int_{B(0, R)}\left|D \mathbf{1}_{U}\right|$ and (2.2)-(2.3) we obtain

$$
\forall R>0 \quad \omega_{N}=R^{1-(N+1)} \int_{B(0, R)}\left|D \mathbf{1}_{U}\right|
$$

which proves that $U$ is a half-space and $U=C$ (by the construction of $C$ ).

But $U=C \equiv P \times \mathbb{R}$ implies that $\partial U$ is a vertical hyperplane, contradicting the fact $\partial U$ is the graph of the function $u$.

Moreover, by the discussions above, we have that the minimal cone $P \times \mathbb{R}$ satisfies $P \times \mathbb{R} \subset C$ and $0 \in \partial(P \times \mathbb{R}) \cap \partial C$. Therefore, $P \times \mathbb{R}$ must coincide with the minimal cone $C$ (cf. [14] or Theorem 2.4 of [9]). This provides the first equality in (1.5) with $P$ a minimal cone singular at the origin.

To conclude we observe that the assumption on the partial derivatives implies the existence of a constant $K>0$ such that for every $j \geq 1$, every $x \in \mathbb{R}^{N}$ and every $\alpha=1, \ldots, k$

$$
u_{j}\left(x+e_{\alpha}\right)-u_{j}(x)=\frac{1}{j} \int_{0}^{j} \frac{\partial u}{\partial x_{\alpha}}\left(j x_{1}, \ldots, j x_{\alpha}+t, \ldots, j x_{N}\right) d t \geq-K,
$$

where $e_{1}, \ldots, e_{k}$ denote the first $k$ vectors of the standard (or natural) basis for $\mathbb{R}^{N}$. Now recall that, up to a subsequence, $\left(u_{j}\right)$ converges almost everywhere to $v$ on $\mathbb{R}^{N}$ (cf. for instance Proposition 16.5 of [8]) so, by letting $j \rightarrow+\infty$ in (2.10), we get that for almost every $x \in P$ the point $x+e_{\alpha}$ also belongs to $P$, for every $\alpha=1, \ldots, k$.

Therefore we have

$$
\forall \alpha=1, \ldots, k \quad P+e_{\alpha} \subseteq P .
$$

By applying Proposition 2.1 we deduce that $P$ is a cylinder in the directions $e_{1}, \ldots, e_{k}$, for otherwise, $P$ would be a half-space of $\mathbb{R}^{N}$ contradicting the fact that $P$ is singular at the origin.

Since $P$ is a cylinder in the directions $e_{1}, \ldots, e_{k}$ we must have $P=\mathbb{R}^{k} \times P^{\prime}$, with $P^{\prime} \subset \mathbb{R}^{N-k}$ minimal (since $P$ is minimal in $\mathbb{R}^{N}$ ) and $P^{\prime}$ singular at the origin of $\mathbb{R}^{N-k}$ (since $P$ is singular at the origin of $\mathbb{R}^{N}$ ). This concludes the proof.

Now we can prove the main result. 
Proof of Theorem 1.1. The function $v(x)=u(x)-u(0)$ is a solution of (1.3) with $v(0)=0$ and with $N-7$ partial derivatives bounded on one side (not necessarily the same).

Up to change the variable $x_{j}$ in $-x_{j}$ (if necessary), we can suppose that $v$ has $N-7$ partial derivatives which are bounded from below. Also, we can assume that those partial derivatives are taken with respect to the first $N-7$ variables. If $v$ were not linear then, by applying Theorem 1.2 with $k=N-7$, we would get

$$
C=P \times \mathbb{R}=\left(\mathbb{R}^{N-7} \times P^{\prime}\right) \times \mathbb{R},
$$

where $P^{\prime} \subset \mathbb{R}^{7}$ would be a minimal cone singular at the origin. But this is is impossible since all the (non-trivial) minimal cones in dimension $n \leq 7$ are half-spaces. This gives that $v$ is a linear function and so $u$ must be affine, as desired.

Finally we state and proof Proposition 2.1 used in the last part of the proof of Theorem 1.2. This result is essentially contained in (the proof of) Lemma 2.3 of [9].

Proposition 2.1. Assume $k \geq 2$ and let $\mathcal{C}$ be a non-trivial minimal cone in $\mathbb{R}^{k}$ such that $\mathcal{C}+v \subseteq \mathcal{C}$, for some $v \in \mathbb{R}^{k} \backslash\{0\}$. Then, either $\mathcal{C}+v \neq \mathcal{C}$ and $\mathcal{C}$ is a half-space or, $\mathcal{C}+v=\mathcal{C}$ and $\mathcal{C}$ is a cylinder in the direction $v$.

Proof. Same proof of Lemma 2.3 of [9].

Acknowledgements: The author thanks E. Valdinoci and L. Mazet for a careful reading of a first version of this article. The author is partially supported by the ERC grant EPSILON (Elliptic Pde's and Symmetry of Interfaces and Layers for Odd Nonlinearities) and by the ERC grant COMPAT (Complex Patterns for Strongly Interacting Dynamical Systems).

\section{References}

[1] F.J.Jr. Almgren, Some interior regularity theorems for minimal surfaces and an extension of Bernstein's theorem. Ann. of Math. (2) 84 (1966), $277-292$.

[2] S. Bernstein, Sur un théorème de géométrie et son application aux équations aux dérivées partielles du type elliptique, Comm. Soc. Math. de Kharkov 2 (15) (1915-1917), 38-45. German translation: Über ein geometrisches Theorem und seine Anwendung auf die partiellen Differentialgleichungen vom elliptischen Typus, Math. Z. 26 (1) (1927), 551-558. 
[3] E. Bombieri, E. De Giorgi and E. Giusti, Minimal cones and the Bernstein problem, Invent. Math. 7 (1969), 243-268.

[4] E. Bombieri, E. De Giorgi and M. Miranda, Una maggiorazione a priori relativa alle ipersuperficie minimali non parametriche, Arch. Rat. Mech. Anal., 32, 1969, 255-267.

[5] E. Bombieri, E. Giusti, Harnack's inequality for elliptic differential equations on minimal surfaces Invent. Math. 15, 1, (1972), 24-46.

[6] E. De Giorgi, Una estensione del teorema di Bernstein. Ann. Scuola Norm. Sup. Pisa (3) 19 (1965), 79-85.

[7] A. Farina, A Bernstein-type result for the minimal surface equation. Ann. Scuola Norm. Sup. Pisa (5) XIV (2015), 1231-1237.

[8] E. Giusti, Minimal surfaces and functions of bounded variation. Monographs in Mathematics, 80. Birkhauser Verlag, Basel, 1984.

[9] E. Gonzalez, U. Massari, and M. Miranda, On minimal cones. Appl. Anal., special issue 65 (1997), 135-143.

[10] E. Hopf, On S. Bernstein's theorem on surfaces $z(x, y)$ of nonpositive curvature. Proc. Amer. Math. Soc. 1, (1950), 80-85.

[11] U. Massari, M. Miranda, Minimal surfaces of codimension one. Notas de Matematica, North Holland, Amsterdam, 1984.

[12] M. Miranda, Un principio di massimo forte per le frontiere minimali. Rend. Seminario Mat. Univ. di Padova, 45 (1971), 355-366.

[13] M. Miranda, Superfici minime illimitate. Ann. Sc. Norm. Sup. Pisa (4) 4 (1977) 313-322.

[14] M. P. Moschen, Principio di massimo forte per le frontiere di misura minima. Ann. Univ. Ferrara Sez. VII (N.S.) 23 (1977), 165-168 (1978).

[15] J. Moser, On Harnack's theorem for elliptic differential equations. Comm. Pure Appl. Math. 14 (1961), 577-591.

[16] J. Simons, Minimal varieties in riemannian manifolds. Ann. of Math. (2) 88 (1968), 62-105. 